\title{
An Accurate Liver Segmentation Method Using Parallel Computing Algorithm
}

\author{
Yousif Mohamed Y. Abdallah \\ Department of Radiological Sciences and medical Imaging, College of Medical Applied Sciences, \\ Majmaah University \\ y.yousif@mu.edu.sa
}

\begin{abstract}
In liver, separating touching objects in an image is one of the more difficult image processing operations. Because of the presence of speckle noise in these images affects edges and fine details which limit the contrast resolution and make diagnostic more difficult. Thus, with using segmentation based algorithm, choice of appropriate segmentation technique type for each circumstance becomes an essential task. The watershed transform is often applied to this problem. The watershed transform finds "catchment basins" and "watershed ridge lines" in an image by treating it as a surface where light pixels are high and dark pixels are low. Segmentation using the watershed transform works well if one can identify, or "mark," foreground objects and background locations. This algorithm was done on twenty-five patients. A watershed transform Algorithm liver segmentation method was proposed in this study. Proposed method is able to determine the liver boundaries accurately. It is able to segment liver and improves radiological analysis and diagnosis.
\end{abstract}

Keywords: Text classification; Semantic Web with weighted idf feature; Expanded query; Fuzzy Semantic Web; Fuzzy Ranking Algorithm.

\section{Introduction}

A rapid sequence of images is acquired without table movement immediately after a bolus intravenous injection of radiographic contrast medium. The rate of enhancement in each pixel within the chosen slice can then be used to determine perfusion. The technique provides a quantifiable display of regional perfusion combined with the high spatial resolution afforded by CT. Computed tomography (CT) involves continuous patient translation during x-ray source rotation and data acquisition. As a result, a volume data set is obtained in a relatively short period of time. For chest or abdominal scanning, an entire examination can be completed in a single breath hold of the patient or in several successive short breath holds[1]. The data volume may be viewed as conventional transaxial images or with multiplanar and threedimensional methods. The authors review the technologic aspects of spiral CT, as well as its advantages, limitations, and current clinical applications. Computed tomography (CT or CAT scan) is a noninvasive diagnostic imaging procedure that uses a combination of $\mathrm{X}$-rays and computer technology to produce horizontal, or axial, images (often called slices) of the body[2]. A CT scan shows detailed images of any part of the body, including the bones, muscles, fat, and organs. CT scans are more detailed than standard $\mathrm{X}$-rays. CT scans may be done with or without "contrast." Contrast refers to a substance taken by mouth

DOI: $10.14738 /$ jbemi.23.1187

Publication Date: 31 $1^{\text {st }}$ May 2015

URL: http://dx.doi.org/10.14738/jbemi. 23.1187 
and/or injected into an intravenous (IV) line that causes the particular organ or tissue under study to be seen more clearly[3][4]. Contrast examinations may require fast manipulation for a certain period of time before the procedure. CT scans of the liver and biliary tract may also be used to visualize placement of needles during biopsies of the liver or during aspiration (withdrawal) of fluid from the area of the liver and/or biliary tract[5]. CT scans of the liver are useful in the diagnosis of specific types of jaundice (yellowing of CT scans of the liver and biliary tract (the liver, gallbladder, and bile ducts) can provide more detailed information about the liver, gallbladder, and related structures than standard X-rays of the abdomen, thus providing more information related to injuries and/or diseases of the liver and biliary tract [6][7]. CT scan of the liver and biliary tract may be performed to assess the liver and/or gallbladder and their related structures for tumors and other lesions, injuries, bleeding, infections, abscesses, unexplained abdominal pain, obstructions, or other conditions, particularly when another type of examination, such as X-rays, physical examination, and ultra sound is not conclusive [8]. A CT scan of the liver may be used to distinguish between obstructive and non-obstructive jaundice. Another use of CT scans of the liver and biliary tract is to provide guidance for biopsies and/or aspiration of tissue from the liver or gallbladder [9]. Image segmentation has been a long-standing problem in computer vision. It is a very difficult problem for general images, which may contain effects such as highlights, shadows, transparency, and object occlusion [10]. Segmentation in the domain of medical imaging has some characteristics that make the segmentation task easier and difficult at the same time. On the one hand, the imaging is narrowly focused on an anatomic region [11]. The imaging context is also well-defined. While context may be present to some extent in segmenting general images (e.g., indoor vs. outdoor, city vs. nature, people vs. animals), it is much more precise in a medical imaging task, where the imaging modality, imaging conditions, and the organ identity is known. In addition, the pose variations are limited, and there is usually prior knowledge of the number of tissues and the Region of Interest (ROI). On the other hand, the images produced in this field are one of the most challenging due to the poor quality of imaging making the anatomical region segmentation from the background very difficult [12][13]. Often the intensity variations alone are not sufficient to distinguish the foreground from the background, and additional cues are required to isolate ROls. Finally, segmentation is often a means to an end in medical imaging [14]. It could be part of a detection process such as tissue detection, or for the purpose of quantification of measures important for diagnosis, such as for example, lesion burden which is the number of pixels/voxels within the lesion regions in the brain[15].

\section{The Existing Ranking Methods}

Separating touching objects in an image is one of the more difficult image processing operations. The watershed transform is often applied to this problem. The watershed transform finds "catchment basins" and "watershed ridge lines" in an image by treating it as a surface where light pixels are high and dark pixels are low. Segmentation using the watershed transform works well if one can identify, or "mark," foreground objects and background locations. Marker-controlled watershed segmentation follows this basic procedure:

1. Computation a segmentation function. This is an image whose dark regions are the objects I was trying to segment.

2. Computation the foreground markers. These are connected blobs of pixels within each of the objects. 
Yousif Mohamed Y. Abdallah; An Accurate Liver Segmentation Method Using Parallel Computing Algorithm. Journal of Biomedical Engineering and Medical Imaging, Volume 2, No 3, June (2015) , pp 15-23

3. Computation the background markers. These are pixels that are not part of any object.

4. Modification of the segmentation function so that it only has minima at the foreground and background marker locations.

5. Compute the watershed transform of the modified segmentation function.

Steps of liver segmentation using MatLab program showed in flowchart below:

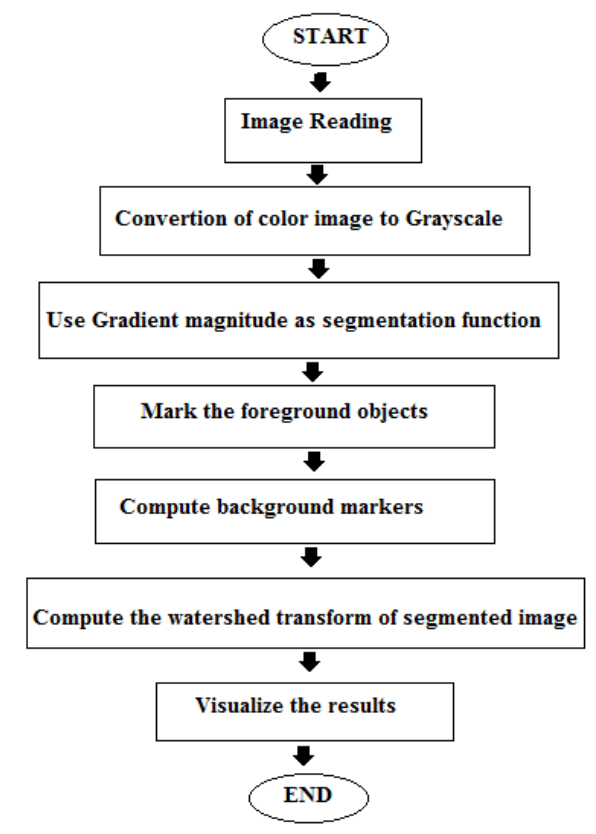

Figure 1. Steps of liver segmentation using MatLab program

\section{The Results}

\section{Experimental study:}

\section{Step 1: Read in the Color Image and Convert it to Grayscale (Figure2)}

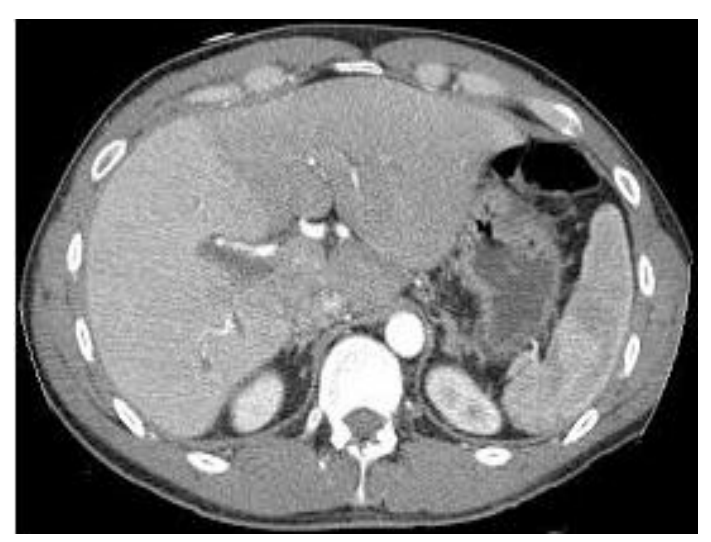

Figure2 Original image used for liver segmentation

\section{Step 2: Use the Gradient Magnitude as the Segmentation Function}

The Sobel edge masks, imfilter, and some simple arithmetic were used to compute the gradient magnitude. The gradient is high at the borders of the objects and low (mostly) inside the objects (Figure3). 


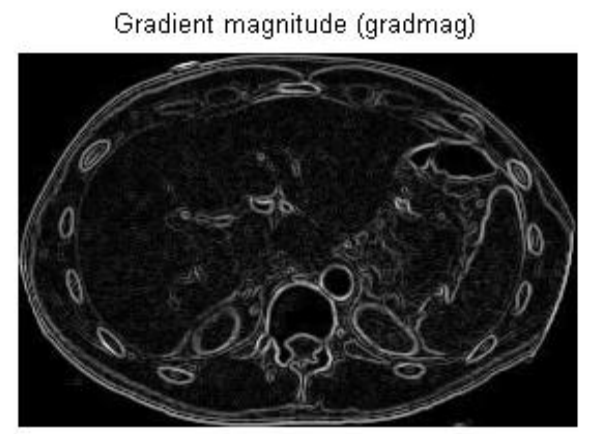

Figure 3. Gradient Magnitude as the Segmentation Function

The image was segmented by using the watershed transform directly on the gradient magnitude (Figure4).

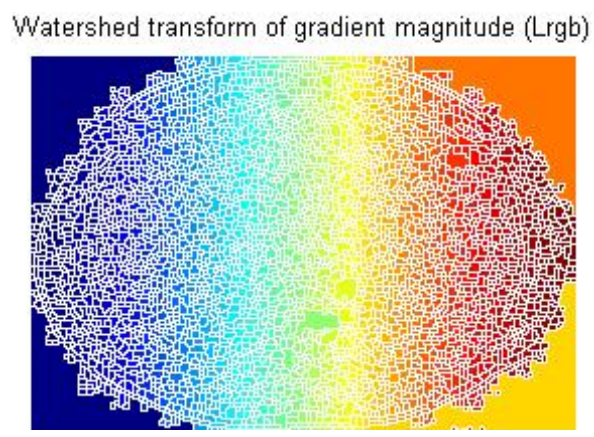

Figure 4. The watershed transform

\section{Step 3: Mark the Foreground Objects}

A variety of procedures could be applied here to find the foreground markers, which must be connected blobs of pixels inside each of the foreground objects. In this study morphological techniques were used and they called "opening-by-reconstruction" and "closing-by-reconstruction" to "clean" up the image. These operations will create flat maxima inside each object that can be located using imregionalmax.

Opening is an erosion followed by a dilation, while opening-by-reconstruction is an erosion followed by a morphological reconstruction (Figure5).

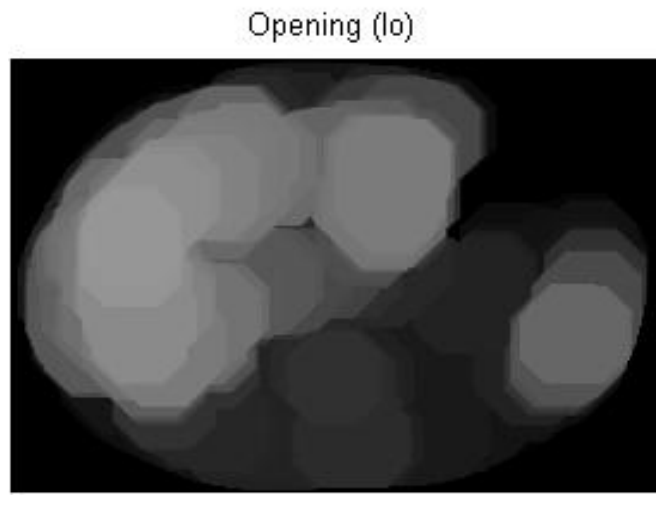

Figure 5. The Opening-by-reconstruction algorithm 
Yousif Mohamed Y. Abdallah; An Accurate Liver Segmentation Method Using Parallel Computing Algorithm. Journal of Biomedical Engineering and Medical Imaging, Volume 2, No 3, June (2015) , pp 15-23

Next the opening-by-reconstruction was computed using imerode and imreconstruct as shown in (Figure6).

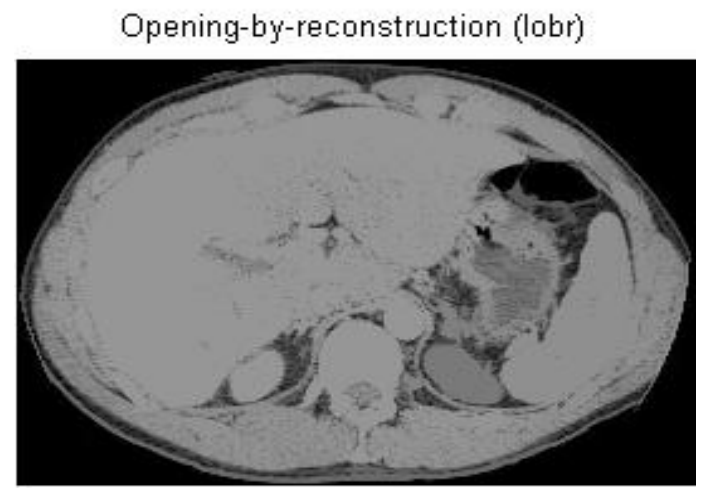

Figure 6. The opening-by-reconstruction was computed using imerode and imreconstruct

Following the opening with a closing can remove the dark spots and stem marks. Compare a regular morphological closing with a closing-by-reconstruction. First imclose code was tried as shown in (Figure6).

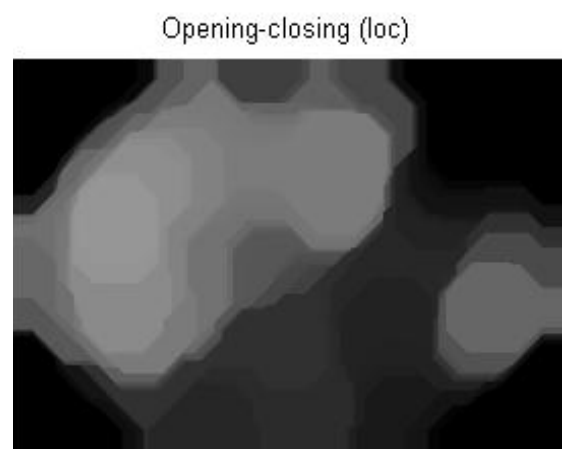

Figure 6. The 'Opening-closing algorithm

The imdilate code was used followed by imreconstruct. The image inputs and output of imreconstruct should complement as shown in (Figure7).

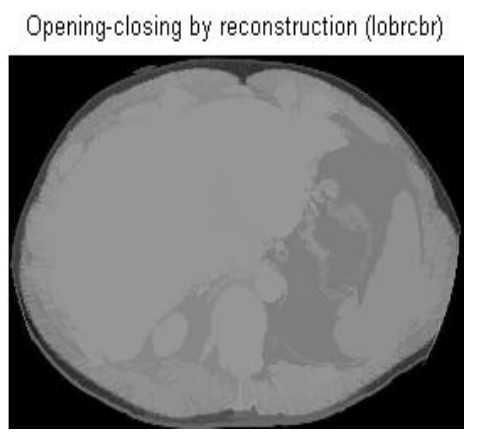

Figure 7. Opening-closing by reconstruction algorithm

When lobrcbr with loc were compared, reconstruction-based opening and closing found more effective than standard opening and closing at removing small blemishes without affecting the overall shapes of 
the objects. Calculate the regional maxima of lobrcbr to obtain good foreground markers as shown in (Figure 8).

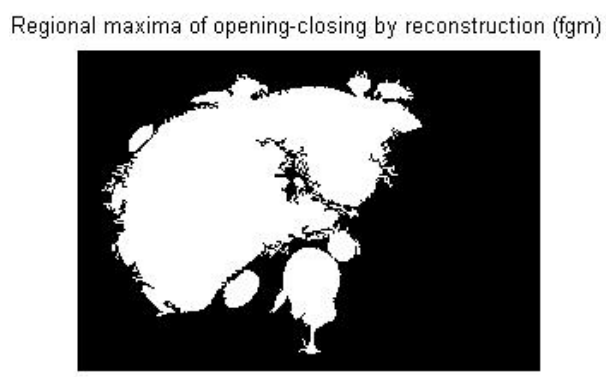

Figure 8. Regional maxima of opening-closing by reconstruction (fgm) filter

To help interpret the result, superimpose the foreground marker image on the original image as shown in (Figure9).

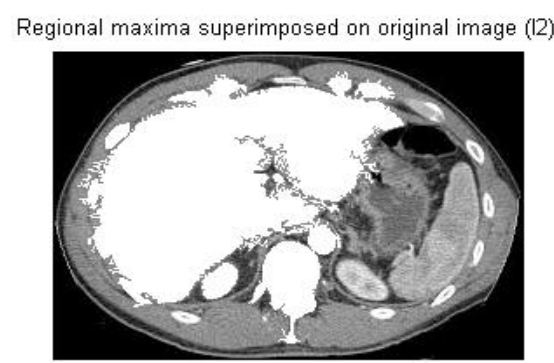

Figure 9. Regional maxima superimposed technique on original image

Some of the mostly-occluded and shadowed objects are not marked, which means that these objects will not be segmented properly in the end result. Also, the foreground markers in some objects go right up to the objects' edge. The edges of the marker blobs should clean and then shrink them a bit. This could be done by a closing followed by an erosion. This procedure tended to leave some stray isolated pixels that must be removed.

This could be done using bwareaopen, which removed all blobs that had less than a certain number of pixels as shown in (Figure10).

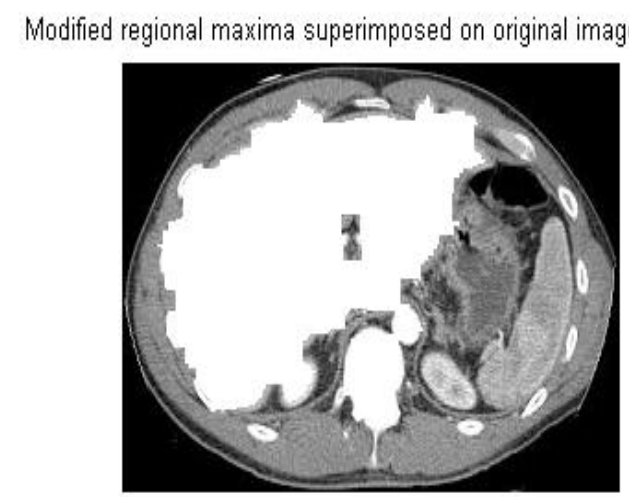

Figure 10. Modified regional maxima superimposed on original image 
Yousif Mohamed Y. Abdallah; An Accurate Liver Segmentation Method Using Parallel Computing Algorithm. Journal of Biomedical Engineering and Medical Imaging, Volume 2, No 3, June (2015) , pp 15-23

\section{Step 4: Compute Background Markers}

Now the background need to be marked. In the cleaned-up image, lobrcbr, the dark pixels belong to the background, a thresholding operation could start with as shown in (Figure11).

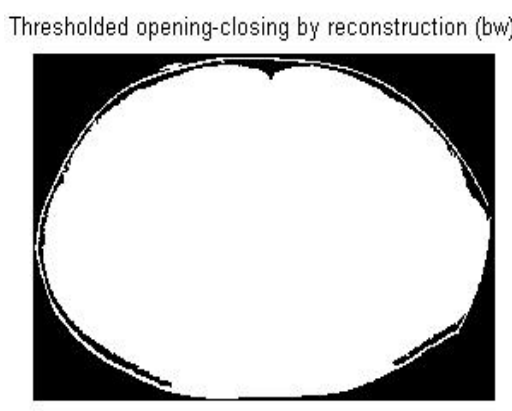

Figure 11. Thresholded opening-closing by reconstruction

The background pixels are in black, but ideally the background markers shouldn't to be too close to the edges of the objects which would segment. the background would "thin" by computing the "skeleton by influence zones", or SKIZ, of the foreground of bw. This can be done by computing the watershed transform of the distance transform of bw, and then looking for the watershed ridge lines ( $D L==0$ ) of the result.

\section{Step 5: Compute the Watershed Transform of the Segmentation Function}

The function imimposemin can be used to modify an image so that it has regional minima only in certain desired locations. Here imimposemin used to modify the gradient magnitude image so that its only regional minima occur at foreground and background marker pixels. Finally I compute the watershedbased segmentation using the following code:

$$
\mathrm{L}=\text { watershed (gradmag2); }
$$

\section{Step 6: Visualize the Result}

One visualization technique is to superimpose the foreground markers, background markers, and segmented object boundaries on the original image. Dilation could use as needed to make certain aspects, such as the object boundaries, more visible. Object boundaries are located where $L==0$ as shown in (Figure 12)

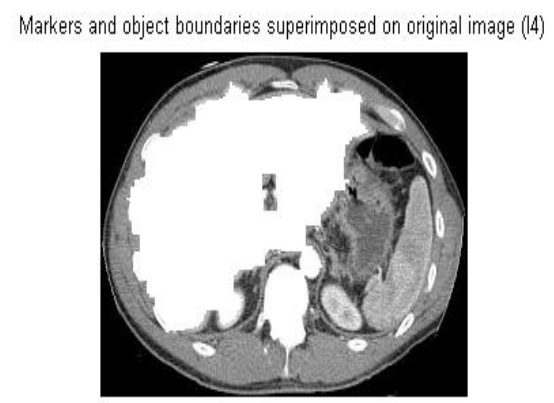

Figure 12. Markers and object boundaries superimposed on original image 


\section{Conclusion}

MatLab is an interactive system whose basic data element is an array that does not require dimensioning. This allows operators to solve many technical computing problems, especially those with matrix and vector formulations, in a fraction of the time it would take to write a program in a scalar non-interactive language such as C or FORTRAN. Magnetic resonance imaging (MRI) has become a common way to study liver. Information provided by medical images has become a vital part of today's patient care. The images generated in medical applications are complex and vary notably from application to application. The main objective of this research was to study an accurate liver segmentation method using a parallel computing algorithm. Computed Tomography images show characteristic information about the physiological properties of the structures-organs. In order to have high quality medical images for reliable diagnosis, the processing of image is necessary. The scope of image processing and analysis applied to medical applications is to improve the quality of the acquired image and extract quantitative information from medical image data in an efficient and accurate way. The main techniques of segmentation used in this study was watershed transform. The results of this technique agreed other author's' results who used different segmentation filtering based on the methods of enhance the computed tomography images. The watershed transform is often applied to solve the contrast and boundaries problem. The watershed transform finds "catchment basins" and "watershed ridge lines" in an image by treating it as a surface where light pixels are high and dark pixels are low. Segmentation using the watershed transform works well if one can identify, or "mark," foreground objects and background locations. This algorithm was done on twenty-five patients. A watershed transform Algorithm liver segmentation method was proposed in this study. Proposed method is able to determine the liver boundaries accurately. It is able to segment liver and improves radiological analysis and diagnosis.

\section{REFERENCES}

[1] Abdallah, Y., Hayder, A., and Wagiallah ,E., Automatic Enhancement of Mammogram using Contrast Algorithm. International Journal of Science and Research (IJSR), 2014. 3(9): p. 18861891.

[2] Abdelwahab, R., and Abdallah, Y., Hayder, A., and Wagiallah ,E., Application of Texture Analysis Algorithm for Data Extraction in Dental X-Ray Images. International Journal of Science and Research (IJSR), 2014. 3(10): p. 1934-1939.

[3] Abdallah, Y., Alkhir, M., Algaddal, A., Improvement of Brain Tumors Detection Using Markers and Boundaries Transform. International Journal of Science and Research (IJSR), 2015. 4(1): p. 2372-2378.

[4] Bidgood, D. \& Horii, S., Introduction to the ACR-NEMA DICOM standard. RadioGraphics, 1992. 12: p. 345-355.

[5] Lehmann, T.M.; Gönner, C. \& Spitzer, K., Survey: Interpolation Methods in Medical Image Processing. IEEE Transactions on Medical Imagin: 1999. 18(11): p. 1049-1075 
Yousif Mohamed Y. Abdallah; An Accurate Liver Segmentation Method Using Parallel Computing Algorithm. Journal of Biomedical Engineering and Medical Imaging, Volume 2, No 3, June (2015) , pp 15-23

[6] Li, G. \& Miller, R.W., Volumetric Image Registration of Multi-modality Images of CT, MRI and PET, Biomedical Imaging. Youxin Mao (Ed.), 2010.

[7] Abdallah, Y., Application of Analysis Approach in Noise Estimation, Using Image Processing Program. Lambert Publishing Press GmbH \& Co. KG, Germany, 2011.

[8] Lyra, M.; Sotiropoulos, M., Lagopati, N. \& Gavrilleli, M. , Quantification of Myocardial Perfusion in 3D SPECT images - Stress/Rest volume differences, Imaging Systems and Techniques (IST), IEEE International Conference 2010: p. 31 - 35

[9] Narasimha-lyer, H., et al., Automatic Identification of Retinal Arteries and Veins From DualWavelength Images Using Structural and Functional Features. Biomedical Engineering, IEEE Transactions, 2007. 54(8): p. 1427-1435.

[10] Abdallah, Y., and Wagiallah, E., Segmentation of Thyroid Scintography Using Edge Detection and Morphology Filters. International Journal of Science and Research (IJSR), 2014. 3(11): p. 2768-2772.

[11] Abdallah, Y., and Hassan, A., Segmentation of Brain in MRI Images Using Watershed-based Technique. International Journal of Science and Research (IJSR), 2015. 4(1): p. 683-688.

[12] Hong, S., et al., Optimal scheduling of tracing computations for real-time vascular landmark extraction from retinal fundus images. Information Technology in Biomedicine, IEEE Transactions on, 2001. 5(1): p. 77-91.

[13] Pinz, A., et al., Mapping the human retina. Medical Imaging, IEEE Transactions on, 1998. 17(4): p. 606-619.

[14] Abdallah, Y., and Yousef R., Augmentation of X-Rays Images using Pixel Intensity Values Adjustments. International Journal of Science and Research (IJSR), 2015. 4(2): p. 2425-2430. 